\title{
Rate of intraoperative problems during sacroiliac screw removal: expect the unexpected
}

\author{
Georg Osterhoff ${ }^{12^{*}}$, Jonas Noser ${ }^{1}$, Kai Sprengel ${ }^{1}$, Hans-Peter Simmen ${ }^{1}$ and Clément M. L. Werner ${ }^{1}$
}

\begin{abstract}
Background: The indications for sacroiliac screw (SI) removal have been under debate. Data on complication rates of SI screw removal is missing in the current literature. The objective of this study was to compare the rate of intraand perioperative problems and complications during SI screw removal to those with SI screw fixation.

Methods: A retrospective observational study with two interventions in the same cohort was performed. Consecutive patients who underwent both sacroiliac screw fixation for an isolated fracture of the pelvic ring and removal of the same implants between November 2008 and September 2015 ( $n=1$; ; age 57.3, SD 16.1 years) were included. Intraoperative technical problems, postoperative complications, duration of surgery, and radiation dose were analysed.

Results: Intraoperative technical problems occurred in 1/19 patients (5\%) during SI screw fixation and in 7/19 cases (37\%) during SI screw removal ( $p=.021$ ). Postoperative complications were seen in 3/19 patients after SI screw fixation and in $1 / 19$ patients after SI screw removal $(p=0.128)$. The surgical time needed per screw was longer for screw removal than for implantation ( $p=.005)$. The amount of radiation used for the whole intervention $(p=.845)$ and per screw $(p=.845)$ did not differ among the two interventions.

Conclusions: Intraoperative technical problems were more frequent with SI screw removal than with SI screw fixation. Most of the intraoperative technical problems in this study were implant-related. They resulted in more surgical time needed per screw removed but similar radiation time.
\end{abstract}

Keywords: Sacroiliac screw fixation, lliosacral screw fixation, Transsacral screw fixation, Implant removal, Pelvic fracture, Sacral fracture, Sacroiliac joint

\section{Background}

Percutaneous sacroiliac (SI) screw fixation has become an established technique for posterior stabilization of pelvic ring injuries [1-6]. Complications associated with this surgical procedure include screw malposition, implant loosening, non-union, and damage to the L5 or sacral nerve roots [2, 7-13]. Revision rates between 2 and 19\% were reported and in most cases the revision procedure was implant removal [2, 7-14].

In their case series on implant removal from the anterior and posterior pelvis, Stuby et al. [15] observed procedurerelated complications in $20 \%$.

\footnotetext{
* Correspondence: georg.osterhoff@medizin.uni-leipzig.de

${ }^{1}$ Department of Trauma, University Hospital Zurich, Rämistrasse 100, 8091 Zurich, Switzerland

2Department of Orthopaedics, Trauma and Plastic Surgery, University Hospital of Leipzig, Liebigstr. 20, 04103 Leipzig, Germany
}

On the topic of SI screw removal itself, there has been published only a small number of case reports and technical notes [16-19]. These stress the challenges associated with the intervention and point on the potential complications.

While implant removal may be well necessary in case of a symptomatic malpositioned screw, other indications for SI screw removal have been under debate. Some authors see an indication for removal only in case of complications $[9,20]$, others recommend implant removal routinely $[7,21]$. Only about a third of the symptomatic patients report sufficient relief of their complaints after the procedure [15]. In addition, many patients ask for removal without being symptomatic.

To the authors' knowledge, data on the complication rates of SI screw removal are underreported in the

(C) The Author(s). 2019 Open Access This article is distributed under the terms of the Creative Commons Attribution 4.0 International License (http://creativecommons.org/licenses/by/4.0/), which permits unrestricted use, distribution, and 
current literature. These would be important order to weigh the potential benefits against the risks associated with this procedure.

The purpose of this study was to compare the rate of intra- and perioperative problems and complications during SI screw removal to those to be expected with their implantation - in one and the same cohort of patients.

\section{Methods}

The protocol of the present study was approved by the local ethics committee (Kantonale Ethik-Kommission Zürich, KEK-ZH-Nr. 2014-0557) and it was assured that patients included into this analysis had not objected to use of their data for research purposes.

A retrospective observational study was conducted. In order to avoid confounding factors as age, gender, co-morbidities, and the individual anatomy of the pelvis, a repeated measures design with two interventions in the same patient was chosen to compare the effect of two interventions (sacroiliac screw fixation and sacroiliac screw removal) on the perioperative outcome in the same patient. Consecutive patients who underwent both sacroiliac screw fixation for an isolated fracture of the pelvic ring and removal of the very same implants between November 2008 and September 2015 were identified $(n=19)$ from a pelvic ring fracture database (total number of patients with SI screw fixation in this period: $n=229)$ and a retrospective chart review was performed. Patients younger than 18 years and those with previous surgery to the pelvis were excluded.

\section{Pelvic screw fixation}

Treatment decisions were based on the fracture classification described by Young \& Burgess [22] using pelvic CT-scans and three-dimensional reconstructions thereof [23]. The decision for operative treatment was made in case of vertical shear (VS, OTA 61-C1) fractures, lateral compression (LC) type II (OTA 61-B2.2) and LC III (OTA 61-B3.2 and C2) fractures and of antero-posterior compression (APC) type III fractures (OTA 61-C1 and C3). For APC II (OTA 61-B1 and B3.1) fractures the indication for surgery was restricted to obese patients or patients that had to be mobilized quickly (i.e. elderly patients). In LC III and APC II and III injuries, anterior fixation was added by the means of plate fixation or internal subcutaneous fixation $[24,25]$. Patients with LC I (OTA 61-B2.1) fractures were operated when non-operative treatment had failed or when a complete sacral fracture in addition with bilateral anterior ring injuries was present [26].

Surgery was performed with the patient supine using cannulated screws $(7.3 \mathrm{~mm}$; DePuy Synthes, Zuchwil, Switzerland) with a washer in the pedicles of S1 and/or
S2 using conventional C-arm fluoroscopy as previously described $[2,4,13]$. In fractures without comminution, threads of $32 \mathrm{~mm}$ were placed in a lag screw fashion; otherwise, fully threaded screws were used.

Postoperatively, patients were allowed to mobilize with $15 \mathrm{~kg}$ partial weight bearing on the affected side for at least 6 weeks.

\section{Implant removal}

Implant removal was performed in a short-term in-hospital setting. Patients with additional anterior implants underwent removal of the SI screws only. In all cases, it was aimed for a complete removal of the screws including washers. Through the scar of the previous surgery, a K-wire was introduced into the sacroiliac screw under $\mathrm{C}$-arm assistance. The screw was then removed sliding a cannulated screw driver over the guide wire. The wire was left in place and served as a guide for either a hooked spoon or Kocher forceps in order to remove the washer. If this technique failed, a Kocher clamp, a nerve hook or an angulated scoop were directed towards the washer using the $\mathrm{C}$-arm in different planes.

\section{Outcome parameter}

Primary outcomes were intraoperative technical problems. These were defined as unexpected events that made additional measures necessary (e.g. opening an additional set of instruments) and prolonged the intervention by 10 or more minutes per screw. Secondary outcomes were postoperative complications during the time of hospitalization, these were categorized according to the system established by Clavien and Dindo [27]. Malpositioned screws that were detected during the initial intervention were revised immediately. Malpositioned screws that were detected postoperatively were defined as a postoperative complication. A symptomatic malpositioned screw was defined as a screw that is not placed within the planned bony canal but breaches the cortical borders of the sacrum and causes pain or loss of motor or sensory function.

Tertiary outcomes were duration of surgery and radiation dose.

\section{Statistical analysis}

Statistical analysis was done by the use of SPSS for windows 22.0 (SPSS, Chicago, Illinois, USA). Ordinal and metric data was compared using non-parametric tests; associations between categorical data were tested using crosstabs and Fisher's Exact test. Metric data was presented as mean with standard deviation (SD). Differences were considered significant for values of $p<0.05$. In case of missing data, comparisons were made only with the data available and this was reported in the results section accordingly. 


\section{Results}

\section{SI screw fixation}

Nineteen patients (age 57.3, SD 16.1 years, range 22 to 86 years; 13 female) were included into the final analysis. The indication for sacroiliac screw fixation were traumatic fractures in 17 cases (Young \& Burgess: 14 LC I, 2 LC II, 1 APC II, 1 combined mechanism) and sacral fragility fractures (2 H-type) in two cases. The total number of screws was 4 screws in 2 patients, 3 screws in 1 patient, 2 screws in 8 patients and 1 screw in 8 patients. Hence, the average number of screws inserted was 1.8 (SD 1.0). Twelve received unilateral and 7 patients a bilateral fixation. In all patients, at least one screw was placed into S1, while 9 patients received an additional screw into S2. In one patient with two bilateral screws in $\mathrm{S} 1$ and two bilateral screws in S2, the screws in S1 were augmented with PMMA cement.

\section{SI screw removal}

Sacroiliac screw removal in the same patients was performed mean 213 days (SD 194, range 2 to 606 days) after implantation of the screws. The indication for screw removal was screw malposition in three cases (see also postoperative complications during hospitalization listed below), asymptomatic screw loosening in five cases, SI joint pain in five cases and a planned pregnancy in one case. In five additional asymptomatic cases, the screws were removed only on the patients' explicit request.

\section{Outcome}

Intraoperative technical problems occurred in 1/19 patients (5\%) during SI screw fixation. The patient had an unexpected accumulation of bowel gas that prevented safe placement of an S2 screw.

Complete SI screw removal including the washers was achieved in all patients. No broken screws were observed. During SI screw removal, additional intra-operative measures were necessary in $7 / 19$ cases $(37 \% ; p=.021$, Fig. 1 ). These included problems due to osseous ingrowth of the washer $(n=4)$, the need for an extended incision due to ingrowth of the screw $(n=2)$, and one case with a worn out screw head. A post-hoc power analysis for the primary outcome (intraoperative technical problems) revealed a power of 1.0 based on an observed odds ratio of 10.5 and assuming a correlation coefficient $(\phi)$ for exposure between matched cases and controls of 0.2 [28].

Postoperative complications during the time of hospitalization were seen in three patients after the initial SI screw fixation procedure and in one patient after SI screw removal. After screw fixation, three patients had to undergo revision for screw malposition. After screw removal, one patient had a urinary tract infection that was successfully treated with antibiotics. When categorized according to the classification described by
Clavien and Dindo, there were no significant differences among the two groups $(p=0.128$, Fig. 2$)$.

While the total duration of surgery was not significantly different $(p=.075)$ among the two groups, the time needed per screw was longer for screw removal than for implantation $(p=.005$, Table 1$)$.

Complete data regarding radiation dose was available in only 13 patients. For these, the amount of radiation used for the whole intervention $(p=.845)$ and per screw $(p=.845)$ did not differ among the two interventions (Table 1).

\section{Discussion}

There has been existing uncertainty on the need and benefit of SI screw removal in cases other than for screw malpositioning or implant failure [7, 9, 15, 20, 21]. The purpose of this study was to evaluate intra- and perioperative problems during SI screw removal and to compare these with the initial procedure of SI screw fixation. In our series of 19 patients, we observed more intraoperative technical problems with SI screw removal than with SI screw fixation. These resulted in more surgical time per screw needed for removal than for implantation.

The rate of intraoperative technical problems was consistent with data from a series on implant removal from the anterior and posterior pelvis, that reported intraoperative problems with the washer or broken screws in 4/ 19 cases with SI screw removal [15].

The postoperative complication rates for SI screw fixation (19\%) seen in our study population were higher than those reported in the literature [7-11]. In contrast to other series, however, our study included only patients who had undergone both SI screw fixation and removal. This may have skewed patient selection towards those with malpositioned screws requiring screw removal. Misplaced screws were classified as postoperative complications if they required surgery only. One could argue that these are intraoperative complications of the index surgery. However, if the surgeon does not identify them during surgery they only become a complication if the patient suffers pain or neurological deficits. Otherwise, they are simply radiographically misplaced screws without clinical relevance.

The number of postoperative complications after SI screw removal was smaller than what would be expected from the small body of literature that reports complication rates of about $20 \%$ with implant removal after pelvic fracture stabilization [15].

It could be shown, that SI screw removal is technically challenging and may require more OR time than screw implantation.

Most of the intraoperative technical problems in this study were implant-related. A potential solution to decrease implant-related intraoperative problems is the use 


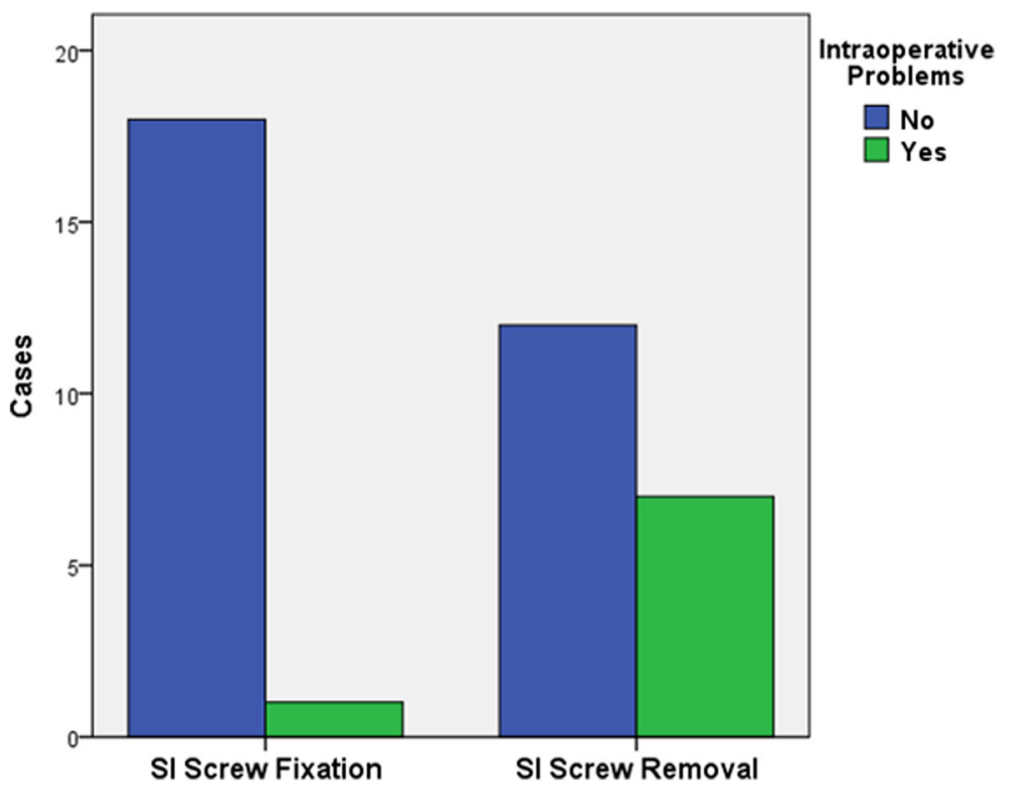

Fig. 1 Intraoperative technical complications

of advanced operative techniques. Described surgical techniques to address the difficulties associated with the retrieval of the washer include endoscopic techniques [18] or the use of a tap of larger diameter in order to achieve interference fit [19]. Broken screws can be removed by using a push screw or drill from the contra-lateral side if this is possible by the screw's orientation [16].

Removing the washer seemed to be the part of the procedure that was most prone to problems. There would have been less intraoperative technical problems and less radiation exposure if we had simply left the washer inside the patients. In fact, without the problems caused by washers, the differences between the two interventions would have been negligible. Just removing the screw and leaving the washer where it is, is a frequent topic that we discuss with the patients preoperatively. However, most of the patients preferred complete implant removal and thus leaving the washer may be not

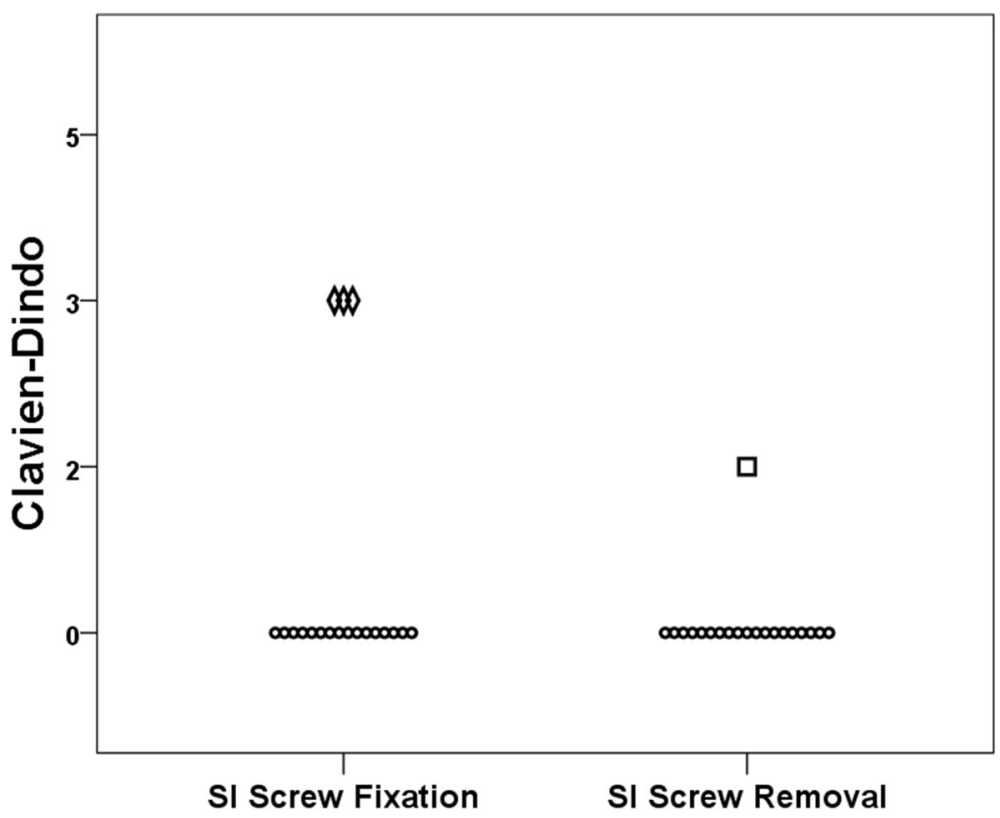

Fig. 2 Postoperative complications. Each symbol represents one patient 
Table 1 Duration of surgery and radiation dose

\begin{tabular}{lllll}
\hline & $N$ & SI Screw Fixation & Removal & $p$ \\
\hline Duration of surgery [min] & 19 & & & \\
$\quad$ total & & $29.0(11.4)$ & $37.6(14.8)$ & .075 \\
$\quad$ per screw & & $15.2(5.3)$ & $21.3(6.3)$ & .005 \\
Radiation dose [mGy] & 13 & & & \\
$\quad$ total & & $27.2(19.3)$ & $27.5(21.2)$ & .845 \\
$\quad$ per screw & & $11.4(6.5)$ & $13.0(8.6)$ & .845 \\
\hline
\end{tabular}

Data reported as mean and standard deviation (in brackets)

min minutes, mGy Milli-Gray

a good option in these cases. In contrast, a prolonged surgical time or even an extended incision with additional surgical risk to remove a washer that is asymptomatic at the preference of the patient might be an even less preferable option.

A different approach to decrease implant-related problems is the development of better implants. While the biomechanical effect of using washers to allow for better compression has been shown [29], their removal obviously is associated with difficulties. One solution could be a washer that is movably mounted to the screw head and, hence, would allow for removal of the screw and the washer as a whole.

The limitations of this study are related to its retrospective study design and the small number of patients included. Even though the matched or repeated measure design of this study allows for ruling out age, gender, co-morbidities, and the individual anatomy of the pelvis as confounding factors, it is not possible to finally conclude that SI screw removal is more prone to complications than SI screw fixation. The time of screw removal ranged from 2 days to almost 2 years. This may have caused a bias with regard to more osseous overgrowth of the screw and washer in patients with late removal. With the numbers available, however, further subgroup analyses were not possible and could be objective of future studies.

The data for radiation exposure was incomplete (13/19). However, radiation exposure was not the primary outcome but chosen as an additional secondary parameter in order to quantify the number of fluoroscopic images taken intraoperatively and to illustrate the surgeon's strain.

Further studies are needed to prove any potential benefit of SI screw removal in patients other than those with complications as symptomatic implant malpositioning. Patients who wish their implants to be removed should meticulously be informed on the risks and benefits of this procedure.

\section{Conclusions}

Intraoperative technical problems were more frequent with SI screw removal than with SI screw fixation. Most of the intraoperative technical problems in this study were implant-related. They resulted in more surgical time needed per screw removed but similar radiation time.

In light of our results, SI screw removal is an intervention with considerable radiation exposure and high rates of intraoperative problems. Even though the complications associated with this procedure rarely have long-term consequences, routine removal cannot be recommended.

Abbreviations

APC: Anterior-posterior compression; CT: Computed tomography; LC: Lateral compression; SD: Standard deviation; SI: Sacroiliac

Acknowledgements

Not applicable.

Funding

There was no external source of funding.

Availability of data and materials

Anonymized source data can be obtained from the corresponding author by email (georg.osterhoff@usz.ch).

\section{Authors' contributions}

JN and CMLW had the idea for this study. GO and CMLW participated in outlining the concept and design. GO and JN did the data acquisition. GO did the statistical analysis and wrote the first draft of the manuscript. JN, KS, HPS, and CMLW revised the final manuscript. All authors have read and approved the manuscript.

\section{Ethics approval and consent to participate}

The protocol of the present study was approved by the local ethics committee (Kantonale Ethik-Kommission Zürich, KEK-ZH-Nr. 2014-0557, and Amendment of November 24, 2015).

The need for individual informed consent was waived by the ethics committee but it was assured that patients included into this analysis had not objected to use of their data for research purposes.

Consent for publication

Not applicable.

\section{Competing interests}

CMLW is a member of the editorial board (Associate Editor) of BMC Surgery. All other authors declare that they have no conflicts of interests.

\section{Publisher's Note}

Springer Nature remains neutral with regard to jurisdictional claims in published maps and institutional affiliations.

Received: 22 October 2018 Accepted: 3 April 2019

Published online: 15 April 2019

\section{References}

1. Matta JM. Indications for anterior fixation of pelvic fractures. Clin Orthop Relat Res. 1996:329:88-96.

2. Osterhoff G, Ossendorf C, Wanner GA, Simmen HP, Werner CM. Percutaneous iliosacral screw fixation in $\mathbf{S 1}$ and $\mathbf{S} 2$ for posterior pelvic ring injuries: technique and perioperative complications. Arch Orthop Trauma Surg. 2010;131:809-13.

3. Farrell EDMD, Gardner MJMD, Krieg JCMD, Chip Routt MLJMD. The upper sacral nerve root tunnel: an anatomic and clinical study. J Orthopaedic Trauma. 2009:23:333-9.

4. Routt ML Jr. Posterior pelvic-ring disruptions: Iliosacral screws. In: Wiss DA, editor. Fractures. Philadelphia: Lippincott Williams \& Wilkins; 2006. p. 649-67.

5. Mears SC, Sutter EG, Wall SJ, Rose DM, Belkoff SM. Biomechanical comparison of three methods of sacral fracture fixation in osteoporotic bone. Spine. 2010;35:E392-5.

6. Tsiridis E, Upadhyay N, Gamie Z, Giannoudis PV. Percutaneous screw fixation for sacral insufficiency fractures: a review of three cases. J Bone Joint Surg Br. 2007;89:1650-3. 
7. Gänsslen A, Hüfner T, Krettek C. Percutaneous iliosacral screw fixation of unstable pelvic injuries by conventional fluoroscopy. Oper Orthop Traumatol. 2006;18:225-44.

8. Hilgert RE, Finn J, Egbers HJ. Technique for percutaneous iliosacral screw insertion with conventional C-arm radiography. Unfallchirurg. 2005;108(954): 6-60.

9. Routt ML Jr, Simonian PT, Mills WJ. Iliosacral screw fixation: early complications of the percutaneous technique. J Orthop Trauma. 1997;11: 584-9.

10. Schweitzer D, Zylberberg A, Cordova M, Gonzalez J. Closed reduction and iliosacral percutaneous fixation of unstable pelvic ring fractures. Injury. 2008; 39:869-74.

11. van den Bosch EWMD, van Zwienen CMAMD, van Vugt ABMD. Fluoroscopic positioning of sacroiliac screws in 88 patients. J Trauma-Injury Infection \& Critical Care. 2002:53:44-8.

12. Peng KT, Huang KC, Chen MC, Li YY, Hsu RW. Percutaneous placement of iliosacral screws for unstable pelvic ring injuries: comparison between one and two C-arm fluoroscopic techniques. J Trauma. 2006;60:602-8.

13. Osterhoff G, Ossendorf C, Wanner GA, Simmen HP, Werner CM. Posterior screw fixation in rotationally unstable pelvic ring injuries. Injury. 2011;42:992-6.

14. Zwingmann J, Konrad G, Mehlhorn AT, Sudkamp NP, Oberst M. Percutaneous iliosacral screw insertion: malpositioning and revision rate of screws with regards to application technique (navigated vs. conventional). J Trauma. 2010;69:1501-6.

15. Stuby FM, Gonser CE, Baron HC, Stockle U, Badke A, Ochs BG. Hardware removal after pelvic ring injury. Unfallchirurg. 2012:115:330-8.

16. El Dafrawy MH, Osgood GM. Retrieval of broken iliosacral screws: the power of a push screw. Injury. 2015;46:1411-6.

17. Weil YA, Nousiainen MT, Helfet DL. Removal of an iliosacral screw entrapping the $L 5$ nerve root after failed posterior pelvic ring fixation: a case report. J Orthop Trauma. 2007;21:414-7.

18. Oberst M, Konrad G, Herget GW, El Tayeh A, Suedkamp NP. Novel endoscopic sacroiliac screw removal technique: reduction of intraoperative radiation exposure. Arch Orthop Trauma Surg. 2014;134:1557-60.

19. Langfitt MK, Best BJ, Carroll EA. A useful tool for retained washer retrieval when removing lliosacral screws. J Surg Orthop Adv. 2013;22:330-2.

20. Abumi K, Saita M, lida T, Kaneda K. Reduction and fixation of sacroiliac joint dislocation by the combined use of $\mathrm{S} 1$ pedicle screws and the Galveston technique. Spine (Phila Pa 1976). 2000;25:1977-83.

21. Culemann U, Tosounidis G, Reilmann H, Pohlemann T. Injury to the pelvic ring. Diagnosis and current possibilities for treatment. Unfallchirurg. 2004; 107:1169-81 quiz 82-3.

22. Burgess AR, Eastridge BJ, Young JW, Ellison TS, Ellison PSJ, Poka A, et al. Pelvic ring disruptions: effective classification system and treatment protocols. J Trauma. 1990;30:848-56.

23. Osterhoff G, Scheyerer MJ, Fritz Y, Bouaicha S, Wanner GA, Simmen HP, et al. Comparing the predictive value of the pelvic ring injury classification systems by tile and by Young and Burgess. Injury. 2014;45:742-7.

24. Scheyerer MJ, Zimmermann SM, Osterhoff G, Tiziani S, Simmen HP, Wanner $\mathrm{GA}$, et al. Anterior subcutaneous internal fixation for treatment of unstable pelvic fractures. BMC research notes. 2014;7:133.

25. Gardner MJ, Mehta S, Mirza A, Ricci WM. Anterior pelvic reduction and fixation using a subcutaneous internal fixator. J Orthop Trauma. 2011;26:314-21.

26. Bruce B, Reilly M, Sims S. OTA highlight paper predicting future displacement of nonoperatively managed lateral compression sacral fractures: can it be done? J Orthop Trauma. 2011;25:523-7.

27. Dindo D, Demartines N, Clavien PA. Classification of surgical complications: a new proposal with evaluation in a cohort of 6336 patients and results of a survey. Ann Surg. 2004;240:205-13.

28. Dupont WD, Plummer WD Jr. Power and sample size calculations. A review and computer program. Control Clin Trials. 1990;11:116-28.

29. Bishop JA, Behn AW, Castillo TN. The biomechanical significance of washer use with screw fixation. J Orthop Trauma. 2014;28:114-7.

\section{Ready to submit your research? Choose BMC and benefit from:}

- fast, convenient online submission

- thorough peer review by experienced researchers in your field

- rapid publication on acceptance

- support for research data, including large and complex data types

- gold Open Access which fosters wider collaboration and increased citations

- maximum visibility for your research: over $100 \mathrm{M}$ website views per year

At BMC, research is always in progress.

Learn more biomedcentral.com/submissions 